\title{
The Nature of Dark Matter in Elliptical Galaxies: Chandra Observations of NGC 4636
}

\author{
M. Loewenstein ${ }^{\mathrm{a} *}$, and R. Mushotzky ${ }^{\mathrm{a}}$ \\ ${ }^{a}$ Laboratory for High Energy Astrophysics, NASA/Goddard Space Flight Center, \\ Code 662, Greenbelt, MD 20771, USA
}

We determine the total enclosed mass profile from 0.7 to $35 \mathrm{kpc}$ in the elliptical galaxy NGC 4636 based on the hot interstellar medium temperature profile measured using the Chandra X-ray Observatory, and other X-ray and optical data. The total mass increases as $r^{1.2}$ to a good approximation over this range in radii, attaining a total of $\sim 1.5 \times 10^{12} \mathrm{M}_{\odot}$ (corresponding to $M_{\mathrm{tot}} / L_{V}=40$ ) at $35 \mathrm{kpc}$. We find that at least half, and as much as $80 \%$, of the mass within the optical half-light radius is non-luminous, implying an exceptionally low baryon fraction in NGC 4636. The large inferred dark matter concentration and central dark matter density, consistent with the upper end of the range expected for standard cold dark matter halos, imply that mechanisms proposed to explain low dark matter densities in less massive galaxies are not effective in elliptical galaxies.

\section{Context}

According to recent estimates, $80-90 \%$ of the matter in the universe is non-baryonic. With the presence of extended dark matter halos in galaxies of all morphological types now wellestablished, attention is focusing on comparing the detailed mass distribution with theoretical predictions of galactic dark halo structure. The shape of the dark matter distribution is determined by the initial density perturbation spectrum, the coupled dynamical evolution of baryonic and non-baryonic constituents, and the nature of the dark matter itself. Therefore, its measurement represents a powerful diagnostic of fundamental astrophysical processes and parameters.

The standard cold dark matter (CDM) model is highly successful in explaining the distribution of mass in the universe on scales ranging from galaxies on up, but is undergoing a critical reexamination - due in large part to its confrontation with measurements of late-type galaxy mass distributions indicating that dark matter is less concentrated than expected. In this work [1], we use a recent Chandra X-ray Observatory observation of NGC 4636 to constrain its dark matter

\footnotetext{
*also with University of Maryland Department of Astronomy
}

distribution, with the goal of testing whether this "concentration crisis" applies to this (early-type) elliptical galaxy.

\subsection{Chandra and Dark Matter Estimation}

Hot, extended distributions of X-ray emitting hot gas in hydrostatic equilibrium are crucial tracers of the gravitational potential in individual elliptical galaxies [2]; gravitational lensing is useful only in a statistical sense for a large sample of galaxies. The physical properties of the hot gas can be accurately measured out to large radii where dark matter dominates and optical techniques are infeasible. The effective resolution of the mass estimate essentially corresponds to that of the gas temperature profile (see below). The unprecedented Chandra angular resolution enables one to do X-ray imaging spectroscopy of the hot ISM on scales comparable to optical studies of the stars for the first time, thus yielding the relative distributions of luminous and dark matter.

\subsection{Modeling Approach}

Given a mass distribution, and the hot gas density distribution obtained from the measured Xray surface brightness distribution, we solve the equation of hydrostatic equilibrium to derive the 
corresponding gas temperature profile. The total mass model consists of three components an $8 \times 10^{7} \mathrm{M}_{\odot}$ central supermassive black hole [3], stars distributed as measured from Hubble Space Telescope and ground-based photometry, and a dark matter density distribution parameterized by an asymptotic slope at the origin $(\zeta)$, a scale radius characterizing the transition to an $r^{-3}$ decline (as determined by the results of numerical simulations) at large radius, and a normalization. For a given $\zeta$, the dark matter scale and normalization, and the stellar mass-to-light ratio, are varied to obtain a match with the composite temperature profile measured by Chandra and the XMM-Newton Observatory (Figure 1).

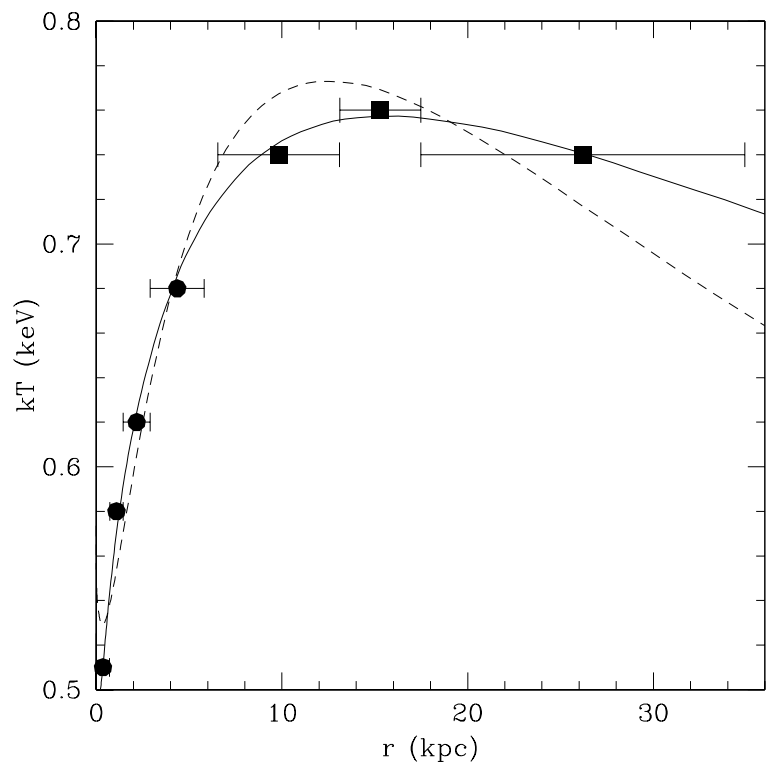

Figure 1. Chandra (filled circles) and XMMNewton (filled squares) temperatures, and profiles for overall best-fit model (which has an $r^{-1}$ dark matter density cusp; solid curve), and bestfit model with dark matter density core (broken curve).

\section{Results}

Models with constant mass-to-light ratios and those where the dark matter density inner slope, $\zeta$, is as steep as the singular isothermal sphere value of -2 are clearly ruled out. We derive accurate constraints on the total mass distribution from $0.7-35 \mathrm{kpc}$ that are robust to the assumed value of $\zeta$. The total mass increases as $r^{1.2}$ (more quickly than the stars) to a good approximation over this range in radii, attaining a total of $\sim 1.5 \times 10^{12} \mathrm{M}_{\odot}$ (corresponding to $M_{\text {tot }} / L_{V}=40$ in solar units) at the outermost point we consider (Figure 2).

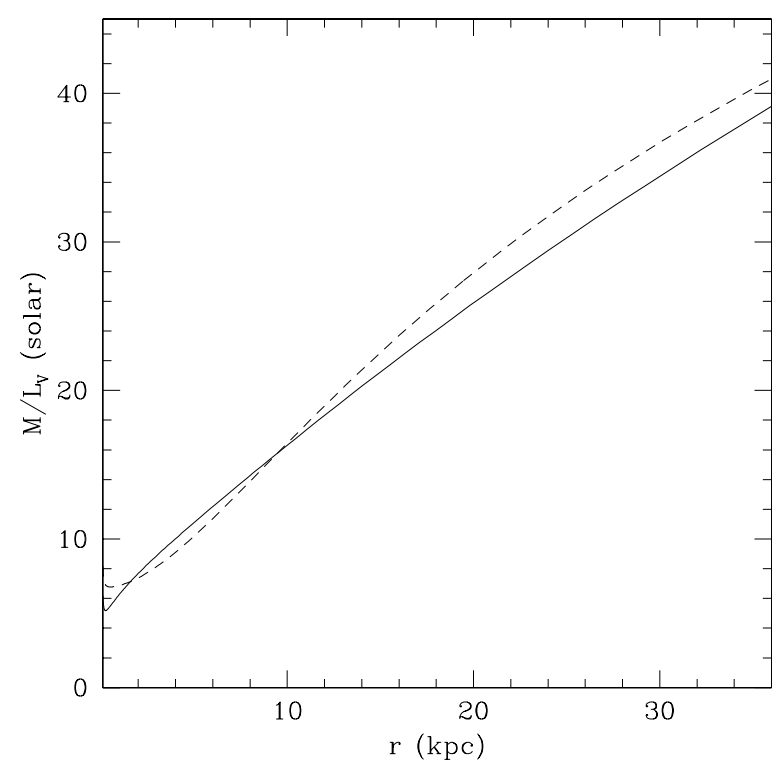

Figure 2. Mass-to-light ratio, in solar units, for the models corresponding to the temperature profiles of Figure 1.

There are degeneracies with respect to the relative distributions of dark and luminous matter. We find acceptable models with dark matter cusps (e.g., $\zeta=1,1.5)$ and with cores $(\zeta=0)$. The former imply lower stellar mass to light ratios (the allowed range: $M_{\text {stars }} / L_{V}=2.4-6.6$, consistent with studies of the stellar population) and, in some cases, the predominance of dark matter all the way into the nucleus of the galaxy. The dark matter fraction within the half-light radius $(\sim 8$ kpc) can be as high as 0.8 and "central" (actually, the average over the inner $700 \mathrm{pc}$ ) dark matter density as high as $4.0 \mathrm{M}_{\odot} \mathrm{pc}^{-3}$; the cored models define lower limits to these quantities of 0.5 and $0.16 \mathrm{M}_{\odot} \mathrm{pc}^{-3}$, respectively. Even the lowest allowed central density is an order of magnitude 
greater than the previously estimated values for other types of galaxies (Figure 3) that precipitated the consideration of alternatives to CDM.

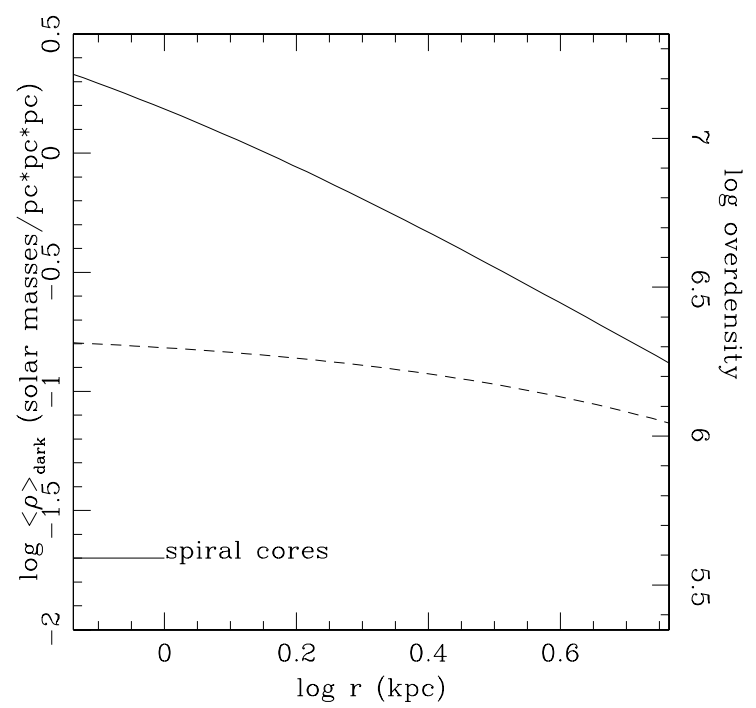

Figure 3. Average enclosed dark matter density distribution corresponding to the temperature profiles of Figure 1. The typical value estimated for spiral (and other) galaxies is indicated.

\section{Implications for the Nature of Dark Matter}

Comparison of non-parametric measures of the dark matter concentration with expectations based on standard CDM numerical simulations indicate that dark matter in NGC 4636 is more compact than average for its mass scale, but within the expected scatter. This is consistent with the findings of statistical studies of the gravitational lensing properties of elliptical galaxies, though it appears that NGC 4636 is unusually dark matter dominated.

Many of the proposed scenarios for reducing the dark matter concentration in late-type galaxies are at odds with our results. It is instructive to consider the two classes of models illustrated in the above Figures from this perspective - those with flat dark matter cores, and those with cuspy dark matter cores (that provide better fits to the X-ray data) interpreted as contracting from initially flat cores due to baryonic infall. For the flat-core models, the high central dark matter mass density and large cores are contrary to the expected scaling relations for selfinteracting dark matter (SIDM) and other models where dark matter structure is driven by dark matter particle interaction. For the cuspy-core models the central phase-space density, conserved during adiabatic contraction, is too high. Alternatively, the velocity-dependent cross section can be fine-tuned so that interactions are ineffective at the higher mass (and velocity) scale of ellipticals; although here too, one may run into phase space difficulties if ellipticals form from mergers of lower mass systems with interaction-induced dark matter cores.

Scenarios where the mechanism for reducing the dark matter concentration becomes less effective with increasing mass scale are not ruled out, since ellipticals such as NGC 4636 represent the most massive galaxies with the deepest potential wells. These include models that invoke warm dark matter, or the expansion of dark matter due to coupling with a powerful protogalactic starburst-driven baryonic outflow. If this transitional mass is indeed on the giant elliptical galaxy scale, cuspy dark matter distributions in galaxy clusters are implied.

Our result that the central dark matter density in NGC 4636 is $>8-200 \times$ the typical value for less massive galaxies contradicts the naive expectations of bottom-up hierarchical clustering where the most massive systems form latest and reflect the relatively low average density in the universe at that epoch. A cold dark matter initial perturbation spectrum that is tilted or otherwise lacking in small-scale power may help explain Ly- $\alpha$ forest data 堛, but likely exacerbates this contradiction.

Recent work on the relation between the merging histories of galaxies and their morphology [5] may be the key to resolving the dark matter concentration problem. A significant dispersion - in dark matter concentration is predicted by numerical simulations - at any given mass range, but particularly on galaxy scales. This results from 
the stochasticity of the merging process, and introduces the following bias. Galaxies of low central dark matter density represent relatively recently formed systems, and/or particularly fragile galaxies that experienced relatively tranquil assembly histories - these may correspond to disk galaxies where low dark matter central densities are indeed measured. Conversely, giant elliptical galaxies such as, or perhaps particularly, NGC 4636 may form at relatively high redshifts and undergo exceptionally prominent merger histories.

\section{Concluding Remarks}

Possible observational inconsistencies of the (non-interacting) CDM paradigm resulted in a renewed scrutiny and a proliferation of alternative models. Careful analysis of the observational situation in light of more detailed, and more deeply understood, theoretical models, as well as the shortcomings of the proposed alternatives seem to indicate that CDM is withstanding these recent challenges to remain the most viable model of structure in the universe (see J. Primack's contribution to this volume). Our results on the high concentration of dark matter in NGC 4636 evidently strengthens the case for CDM, while introducing other puzzles - such as why NGC 4636 seems to be exceptionally dark matter dominated. Although one must exercise caution in generalizing from our results, the most stringent constraints on alternative models for dark halo structure, that presumably universally apply, emerge from studying such a, possibly extreme, system. Results, in progress, of similar investigations for other galaxies (e.g., NGC 1399, NGC 4472) are anticipated with great interest.

\section{REFERENCES}

1. Loewenstein, M. and Mushotzky, R. F., Astrophysical Journal, submitted.

2. Loewenstein, M. and White, R. E., III, Astrophysical Journal, 518 (1999), 50.

3. Merritt, D. and Ferrarese, L., Monthly Notices of the Royal Astronomical Society, 320 (2001), L30.
4. Alam, S. M. K., Bullock, J. S., and Weinberg, D. H., Astrophysical Journal, submitted.

5. Wechsler, R. H., Bullock, J. S., Primack, J. R., Kravtsov, A., and Dekel, A., Astrophysical Journal, 569 (2002), 52. 